Ann. Zootech., I967, 16 (3), 29I-300.

\title{
FISSURE PALATINE HÉRÉDITAIRE ASSOCIÉE A L'ANKYLOSE DES MEMBRES DANS LA RACE CHAROLAISE
}

\author{
J.-J. LALVERG.TE et P.-C. BLIN \\ Station centrale de Génétique animale, \\ Centre national de Recherches zootechniques, 78 -Jouy-en-Josas; \\ Laboratoire d'Anatomie comparée, École nationale vétérinaire, 94 -Maisons - Alfort \\ Institut national de la recherche agronomique
}

\section{SOMMAIRE}

En race charolaise, des cas de fissure palatine associće à l'ankylose symétrique des genoux, des jarrets et des boulets antérieurs ont été signalés dans les deux sexes.

Incapables de se tenir debout et de téter, les veaux anomaliques, condammés à mourir d'inanition à plus ou moins longue échéance, sont, le plus souvent, sacrifiés par l'éleveur.

L'interprétation du déterminisme héréditaire de ces anomalies associées par un facteur autosomal récessif simple ne donne pas entièrement satisfaction, pour autant çue les exceptions relevées ne sont pas dues à des erreurs de paternité, asse\% fréquentes en race charolaise. I.es auteurs donnent la préférence, pour l'instant, ì une hypothèse faisant intervenir un gène dominant ì pénétrance incomplète. La pénétrance serait voisine de $20 \%$. 100 che\% les hétérozygotes.

On sait, depuis longtemps, qu'il apparaît sporadiquement en France, dans la race charolaise, une anomalie qui semble héréditaire : la fissure palatine.

Nous eûmes l'occasion d'apprendre que cette anomalie s'accompagnait souvent de l'ankylose de certaines articulations des membres $\left({ }^{1}\right)$ et, plus récemment, nous avons pu étudier un sujet qui présentait ces malfornations. La description anatomique que nous en avons faite, ainsi que les déductions génétiques résultant de l'analyse d'observations provenant de deux élevages où est pratiquée la monte naturelle et de deux centres d'insémination artificielle, nous autorisent à présenter cette note préliminaire.

(1) Cette information, itayce de domées nénétiques, nous a été foumie par MII. I) nique de contrôle de la descendance, Gúzzesisec du Centre d'insémination de l'Allier, DE Soras, ćleveur du Cantal. L'animal qui fait l'objet de cette description nous a été confié par M. Merik, ancien technicien à I'I. N. R. A. 
L'animal examiné (1), de sexe mâle, était né à terme chez M. BoRd à Saint-Martin-de-Saint-Vaury (Creuse), le 27 juin I966; il pesait $32 \mathrm{~kg}$ à la naissance ; le vêlage et la délivrance s'étaient effectués normalement.

Ce veau (fig. I), frappé d'atrophie musculaire, était incapable de se tenir debout et même de remuer les membres; il reposait sur le flanc et haletait. Les genoux, les jarrets et les boulets antérieurs étaient ankylosés ; par contre, les autres articulations étaient mobiles, surtout les articulations coxo-fémorales qui offraient une grande laxité, au point qu'on pouvait avec facilité ramener sur le dos les onglons postérieurs jusqu'à leur faire toucher la tête. Frappé de dyspnée et d'insuffisance cardiaque, le veau mourut à l'âge de deux jours, sans avoir pu téter.

\section{I. - ÉTUDE ANATOMIQUE DU VEAU 6 I 40}

Les anomalies relevées portent sur le plafond de la cavité buccale ainsi que sur certaines articulations des membres. Le palais offre une fissure médiane complète prolongée en arrière sur le voile du palais (fissure vélo-palatine médiane totale).

Les membres se caractérisent par l'ankylose symétrique de certaines articulations : le genou et le boulet au membre thoracique, le jarret et le boulet au membre pelvien.

Ces articulations présentent des modifications angulaires, dues essentiellement à la rétraction de certains tendons. Les articulations proximales des membres (épaule, coude, articulation coxo-fémorale, grasset) sont normales mais, contrairement aux articulations ankylosées, les ligaments qui les composent sont lâches, étirés, d'où une mobilité plus grande que d'ordinaire, en particulier, dans le sens de l'extension et de la flexion.

\section{A - Fissure palatine}

La fissure palatine a une quinzaine de centimètres de longueur; les crêtes palatines sont entièrement disjointes sur le plan médian ; la brèche est large de I centimètre entre les premières crêtes; elle atteint $2,3 \mathrm{~cm}$ à hauteur de la cinquième, 3 centimètres au niveau de la septième; elle se rétrécit de nouveau en arrière et mesure moins de 2 centimètres de largeur en région postérieure du palais (fig 2).

En partie orale, la papille incisive, de forme hexagonale, occupe une position normale mais apparaît isolée.

La cloison nasale s'enfonce en mortaise profonde dans le vomer (fig. 3) et n'est pas coalescente avec le palais.

Ira muqueuse pituitaire est très épaisse en région inférieure des faces de cette cloison ; elle constitue, à la limite du tiers antérieur et du tiers moyen du bord ventral du septum, un léger relief médian flanqué de chaque côté par un sillon bien marqué (ce détail est visible sur les fig. 2 et 3 ).

En arrière, la cloison médiane diminue progressivement de hauteur ; son bord inférieur est large, ogival sur la coupe segmentale.

Les cornets naso- et maxillo-turbinaux ont une disposition et une conformation normales.

(1) Nous l'avons enregistré sous le matricule 6r \&o. 


\section{$\mathrm{B}$ - Anomalies des membres}

Elles intéressent, de chaque côté, les articulations du genou, du jarret et du boulet antérieur.

Nous en avons fait un examen radiologique, une description anatomique et une étude goniométrique ; les faits essentiels relevés sont les suivants :

\section{Examen radiologique.}

Les clichés relatifs aux boulets antérieurs montrent un certain degré de fermeture vers l'arrière de ces articulations ainsi que le déplacement vers l'avant des grands sésamoïdes qui semblent "enfoncés " dans 1'angle postérieur métacarpo-phalangien.

Plus intéressants sont les clichés qui se rapportent aux articulations carpiennes et tarsiennes. Les os du genou, sans perdre leurs connexions, subissent des modifications d'orientation; il en résulte une ouverture vers l'avant des interlignes radioet médio-carpiens ; le déplacement en avant et en bas du capitato-trapézoïde est particulièrement net (fig. 4) ; notons que le contour du pisiforme est mal défini.

En ce qui concerne le jarret, le talus, volumineux, apparaît comprimé et légèrement coudé dorso-ventralement ; les os de la deuxième rangée tarsienne sont amincis, aplatis de haut en bas ( $I^{\prime}$, portion scaphoïdienne du scapho-cuboïde et 2 , grand cunéiforme, fig. 5).

\section{Description anatomique.}

Nous avons disséqué préalablement les muscles et les tendons qui intéressent l'extrémité distale de chaque membre ; nous avons observé le " raccourcissement" des tendons phalangiens au membre thoracique et des tendons des muscles jambiers antérieurs, au membre pelvien. Dans le premier cas, le raccourcissement tendineux semblait entraîner la flexion du genou et du boulet antérieur, dans le second cas, une légère fermeture du jarret vers l'avant.

Quant aux malformations ostéologiques. elles ne portent que sur le carpe et sur le tarse.

La première rangée des os du carpe ne présente que trois pièces : le pisiforme, sphéroïde, de volume réduit, de consistance molle, est enclavé entre l'extrémité inférieure de 1'ulna et le pyramidal; le pyramidal est incomplètement soudé en région palmaire au semi-lunaire ; le semi-lunaire et le scaphoïde se soudent complètement l'un à l'autre et forment un massif unitaire.

La deuxième rangée des os du carpe comprend les deux pièces habituelles; 1'os crochu et le capitato-trapézoïde; ces pièces sont aplaties dorso-ventralement en particulier le capitato-trapézoïde (son diamètre vertical est de $7 \mathrm{~mm}$ seulement, en région moyenne).

Les soudures osseuses observées dans le carpe expliquent en partie l'ankylose du genou.

L'étude ostéologique du jarret a montré que le calcaneus est plus massif que normalement, que son sommet est plus bas et légèrement rejeté vers l'avant; quant au talus, il est comprimé dorso-ventralement et faiblement coudé vers l'avant, fait qui corrobore l'examen radiologique ; calcaneus et talus se soudent incomplètement l'un à l'autre par leurs faces adjacentes. 


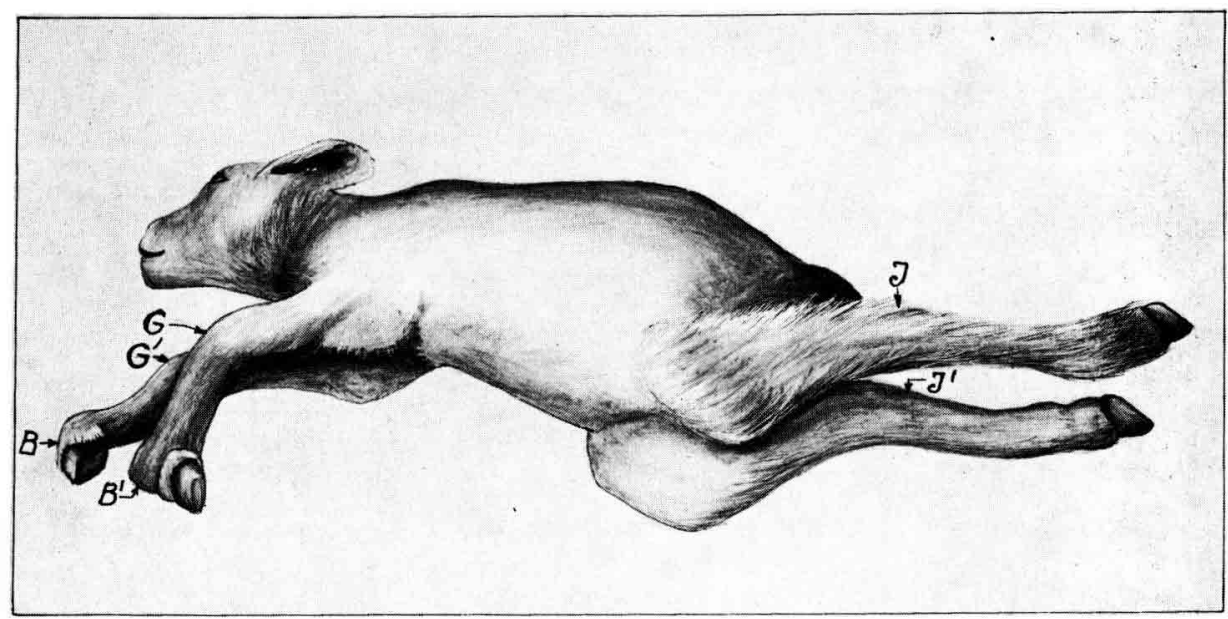

FIG. I. - Extérieur du veau $n^{\circ} 61+0$

(dessin effectué par $\mathrm{XI}^{\mathrm{me}} \mathrm{M}$. Yankovitcil d'après une photographie de l'animal)

Les articulations du genou $\left(G\right.$ et $\left.G^{\prime}\right)$ et du boulet antérieur ( $\left(B\right.$ et $\left.B^{\prime}\right)$ sont anormalement héchies; les membres pelviens sont rejetés vers l'arrière, en raison de la laxité des articulations coxo-fémorales et fémoro-tibio-rotuliennes ; les jarrets $\left(\mathrm{J}\right.$ et $\mathrm{J}^{\prime}$ ) sont légèrement fléchis (ce détail n'apparaît pas nettement sur le dessin).

Fig. 2. - Fissure viélo-palatine médiane complète chez le zeau $n^{0} 6110$

I - l'apille incisive,

$z, 2^{\prime}$ - Crêtes palatines,

3 - - Septum nasal,

$4,4^{\prime}$ - Fosses nasales,

$5,5^{\prime},-$ - Voile du palais (fissuré),

6,6, - P'apilles odontoïdes de la muqueuse jugale.

Frc. 3. - Schima de la conpe segmentale des fosses nasales du zean nu ti140

(niveau de la dixiome paire de (rêtes palatines)

$2,2^{\prime}$ - (rétes palatines (sectionnćs latéralement),

3 , - Mluqueuse septale,

$4, t^{\prime}$ - - Fosses nasaies,

7 - Vomer,

8 - Cloison nasiale,

$9,9^{\prime}$ - Maxillaires,

, C. C.anes du comet maxillo-turbinal,

$\mathrm{C}_{\mathrm{I}}$ - Cornet naso-turbinal. 


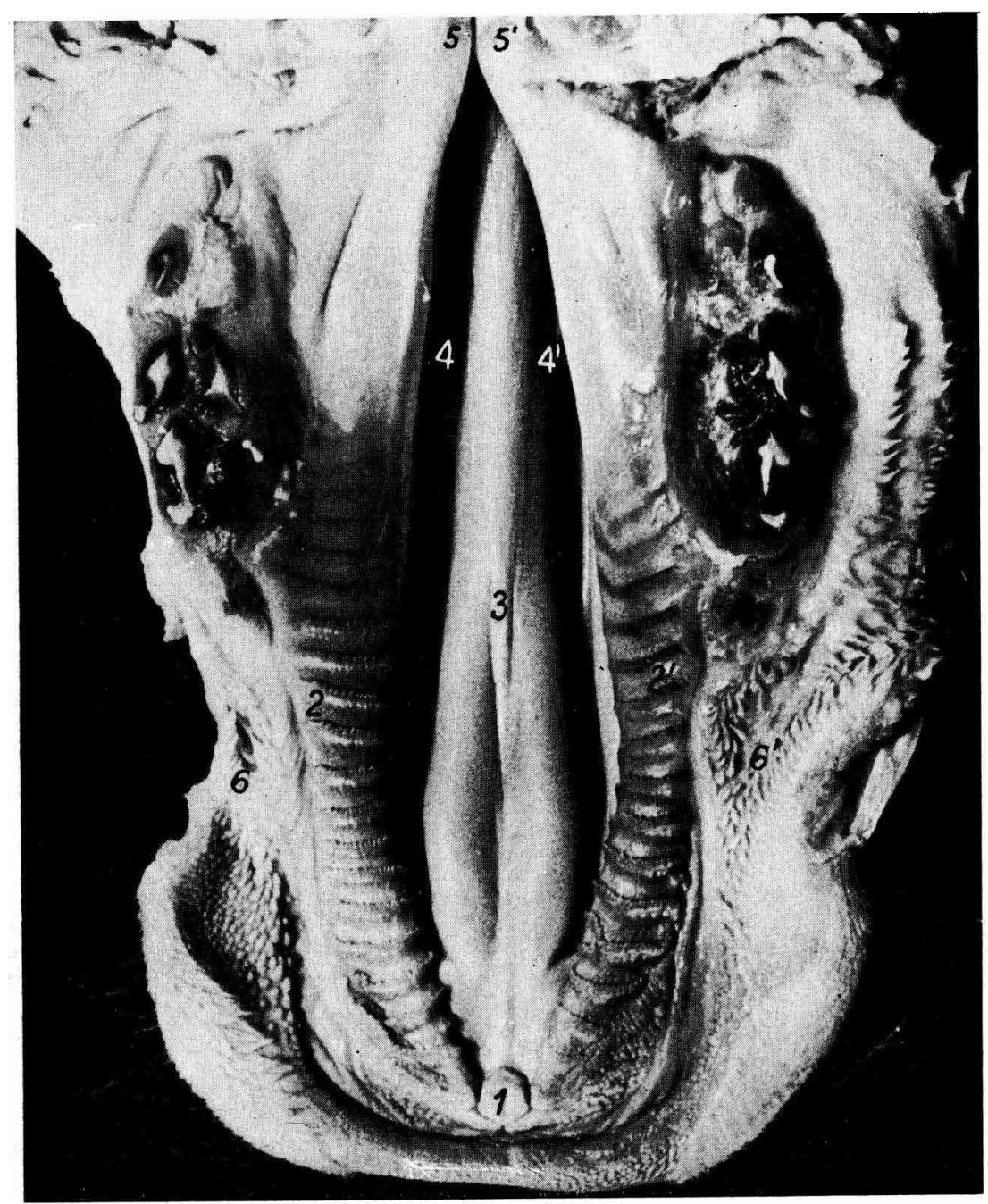

FIG. 2.

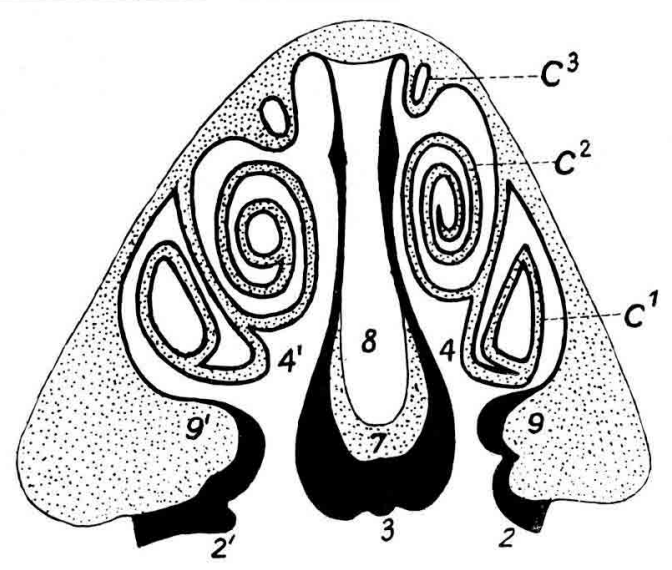

Fis. 3. 


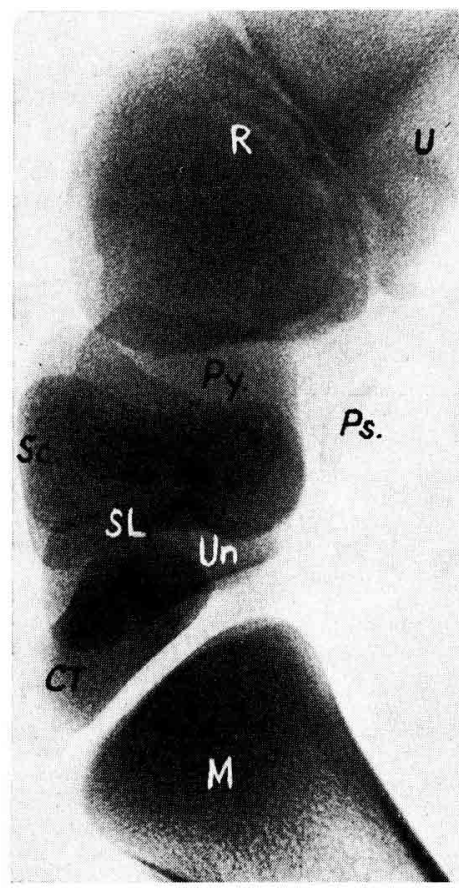

FIG. 4

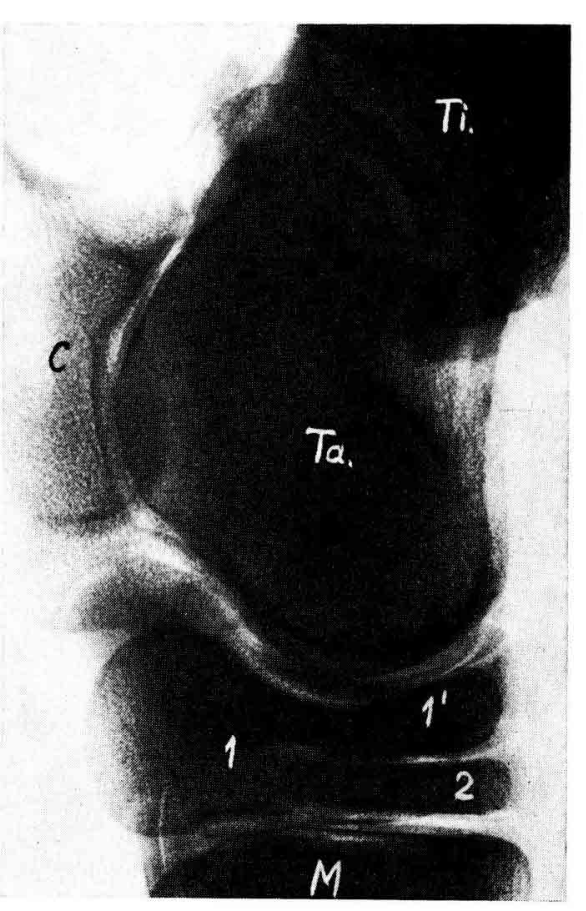

FIG. 5

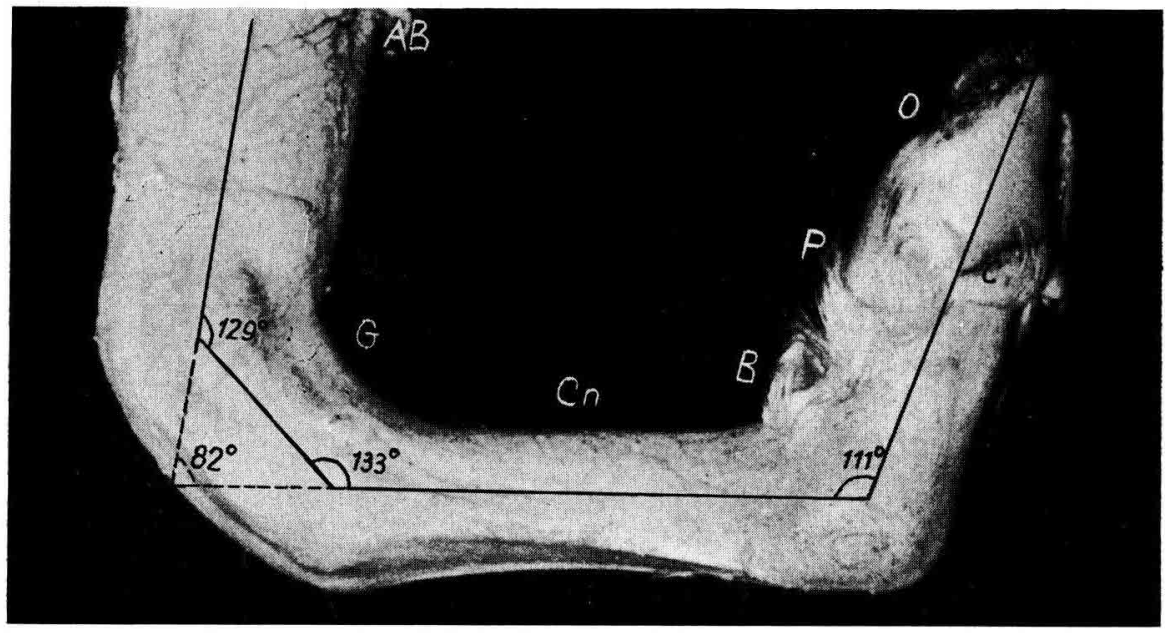

lifi. 6

FIG. 4. - Cliche radiographique du genou gauche (neau $\left.n^{\circ} 6110\right)$

$\mathrm{R}$ : radius; [i : ulna; $\mathrm{I}$ 's : pisiforme; Py : pyramidal;

SL. : semi-lunaire; So: scaphoïde ; Ln : unciforme:

(T: capitato-trapézoïde; $\mathrm{II}:$ métacarpe.

Fis. 5. - Cliché raliographique the larrel ganche (vean $\left.n^{\circ} 6140\right)$

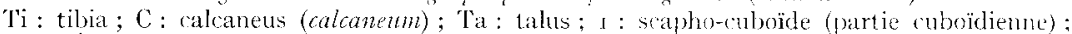

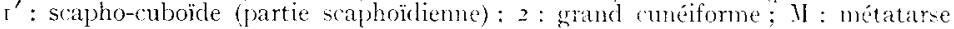

Fic. 6. - Détermination des angles radio-carpien, arpi-métacarpien el métacurpo-phalungien Membre thoracique sauche (veau $n^{\circ}$ 6r

AB : avant-bras ; $G$ : genou : Cu : canon : 13 : boulot :

$P$ : paturon; ( : couronne; 0 : omglon. 
Les os de la deuxième rangée tarsienne sont amincis ; le scapho-cuboïde n'offre pas de saillie postéro-interne répondant au talus. Le petit cunéiforme ne se distingue pas du grand cunéiforme. Les soudures osseuses observées dans le tarse, même partielles, permettent de comprendre également l'ankylose de cette articulation chez le sujet examiné.

3. Étude gonionétrique.

Les mesures d'angles effectuées sur la coupe sagittale des articulations du veau $n^{\circ} 6$ I 40 concrétisent les faits que nous venons d'avancer; elles sont consignées dans le tableau $\mathrm{I}$ et sont illustrées par la figure 6 où nous les avons reportées sur un cliché photographique de l'un des membres antérieurs.

\section{TABI.EAU I}

Valeurs angulaires des divers rayons constituant les articulations ankylosies du thau anormal $n^{0}$ 61.40 comparies à celles que l'on observe che's des zeaux nomaux (dans a dernier cas, l'étude gonioncitrique a étr' fate d'après des clichés radiographiques réalisés sur l'animal deboul)

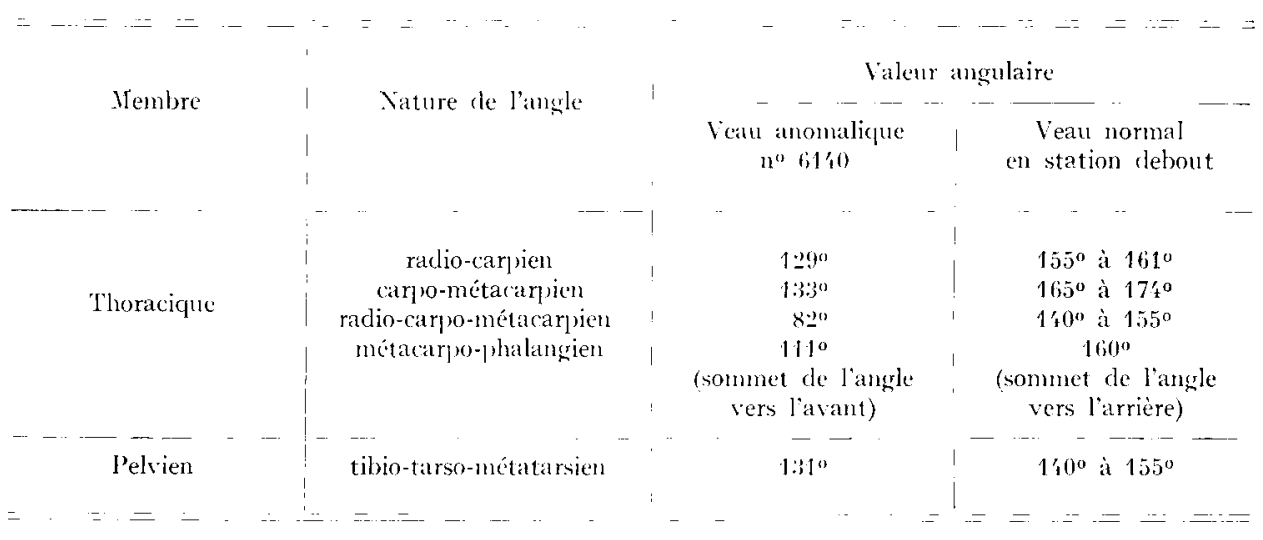

$$
\mathrm{C}-\text { Remarque }
$$

Les deux anomalies, fissure palatine et ankylose des membres, ont été souvent décrites chez les bovins indépendamment l'une de l'autre (KocH et coll,, I957), mais STANG (1940) est le seul à signaler une anomalie héréditaire dans laquelle ces deux défauts sont associés; encore doit-on préciser que dans les cas qu'il présente - il s'agit d'animaux de la race P'ie noire d'Allemagne - l'ankylose était plus généralisée que dans ceux que nous relatons ; elle nécessitait fréquemment une embryotomie.

\section{II. - ANALYSES DES RENSFIGNEMENTS FOURNIS PAR LES ÉLEVAGES}

\section{A - Élevage Bord, à Saint-1Lartin-de-Saint-Vaury (Creuse)}

'Trois veaux ont été examinés en 1965 et I966 et présentaient une ankylose des membres comparable à celle du veau que nous venons d'étudier. Malheureusement, ils furent tous sacrifiés, peu après la naissance, par l'éleveur, qui omit d'examiner le palais et de noter le sexe des animaux. 


\section{B - Elevage de Solan, à Leybros par Ytrac (Cantal)}

Trois veaux anormaux, un mâle et deux femelles naquirent en I964 et I965; deux étaient vivants à la naissance, l'autre, né avant terme, n'a pas survécu. Les animaux ne pouvaient se tenir debout ; seules les articulations coxo-fémorales étaient très mobiles ; dans un cas, une ankylose des boulets fut remarquée par l'éleveur. L'un des veaux ne vécut huit jours que grâce à des soins constants car il rejetait le lait par les naseaux en raison de l'existence d'une fissure palatine. La cavité buccale des deux autres sujets anormaux, dont un né vivant et sacrifié par l'éleveur peu après la naissance, ne fut pas examinée.

\section{C - Centre d'insémination artificielle de l'Allier}

Lin I962 et I963, le technicien, qui avait systématiquement examiné la cavité buccale des veaux anormaux, observa Io cas de fissure palatine ( 6 mâles, 4 femelles) ; chaque fois l'anomalie était accompagnée de malformations des menbres. Malheureusement, les renseignements qu'il donne manquent de rigueur scientifique ; c'est ainsi qu'il parle de : "membres disloqués, difformes, pattes retournées, articulation soudées "...

\section{$\mathrm{D}$ - Centre d'insémination artificielle de la Saône-et-Loire}

Èn rg66, une enquête auprès des éleveurs permit cle signaler 7 cas d'anomalies des membres ( 3 mâles, 4 femelles) chez des animaux qui furent rapidement éliminés de l'élevage. Quatre d'entre eux présentaient une fissure palatine ; le palais des trois autres ne fut pas examiné.

\section{$\mathrm{E}$ - Remarques}

Malgré leur laconisme et leur imprécision, les données fournies par les élevages et les centres d'insémination indiquent que les anomalies en question intéressent à peu près également les deux sexes (sur I 5 cas sùrs, 7 mâles et 8 femelles) ; d'autre part, l'association "fissure palatine-ankylose " apparaît tout à fait constante ; enfin, dernière remarque, elle n'est pas directement létale; le veau ne succombe qu'en raison des difficultés qu'il a à s'alimenter.

\section{III. - ÉTUDE GÉNÉTIQUE}

\section{$A$ - Les données}

\section{I. Élevage Bord.}

'Trois des sujets anormaux descendaient du taureau Uranus et le quatrième de Rhode-Island. I)ans trois cas, nous connaissions également la généalogie de la mère. Ces animaux n'avaient pas d'ancêtres communs avant $1 \mathrm{a} 4^{\mathrm{e}}$ génération, et leur coefficient de consanguinité (Selon MALLCOT, I948) était inférieur à 0,004. 
2. Élevage de Solan.

Les trois veaux anormaux observés avaient tous le même père : un taureau charolais provenant de la région de Decize (Nièvre). Les vaches auxquelles cet animal fut accouplé étaient issues de troupeaux différant par leur origine de celui du mâle. Le taureau engendra 34 descendants.

\section{Centre d'insémination artificielle de l'Allier.}

Les veaux anomaliques furent observés dans la descendance de trois taureaux de testage : Quatrain (8 cas), Quercy (I cas), Quevelleur (I cas) ; l'enquête portait sur une moyenne de roo veaux par taureau. On signale également d'autres taureaux qui engendrèrent des veaux à fissure palatine : Majeur, Energique, Gardian; les deux derniers cités figurent parmi les grand-pères maternels des anormaux de Quatrain.

\section{Centre d'insémination artificielle de Sâ̂ne-et-Loire.}

Il s'agit de la descendance de taureaux de service et on ne connaît pas les grands-pères maternels. Sur une centaine de veaux examinés par taureau on a relevé cinq cas d'anomalie dans la descendance de Quaker et un cas dans la descendance de Sphynx.

\section{$\mathrm{B}$ - Discussion}

Les données du Centre de l'Allier pourraient s'accorder avec une hypothèse de récessivité, car les grands-pères paternels des animaux issus de Quatrain étaient, dans 4 cas sur 5 lorsqu'ils sont connus, des taureaux ayant eux-mêmes engendré des anormaux.

Cependant, l'hypothèse de récessivité simple explique mal le phénomène observé dans les élevages Bord et de Solan où l'on n'a pu établir de relations entre le père et la mère des anormaux. De même, on peut se demander pourquoi on rencontre, dans les centres, à côté de taureaux comme Quatrain et Quaker, dûment responsables de l'apparition de veaux anomaliques, plusieurs autres taureaux qui engendrent occasionnellement un anormal.

Ces faits apparemment contradictoires pourraient s'expliquer dans quelques cas par des erreurs de paternité, assez fréquentes en race charolaise ; si, néanmoins, certains s'avéraient exacts 1'hypothèse génétique la plus logique serait d'attribuer au gène d'anomalie, outre une action à l'état homozygote, un effet dominant à pénétrance incomplète. Les sujets anormaux apparaissant par hasard dans la descendance d'un taureau non porteur du gène d'anomalie seraient issus de vaches hétérozygotes normal overlap $\left(^{1}\right)$. La pénétrance chez les hétérozygotes serait d'environ $20 \mathrm{p}$. Ioo ( 3 cas sur 34 dans la descendance du taureau de M. de Solan).

Afin de vérifier l'action récessive du gène il serait intéressant de procéder à des croisements entre un taureau réputé porteur et des vaches charolaises ayant donné au moins un anormal.

Pour mettre en évidence le caractère de dominance il y aurait lieu d'envisager des accouplements entre taureau réputé porteur et vaches d'une autre race dans laquelle l'anomalie n'existe pas.

$$
\text { Ręu pour publication en mai } 1967 .
$$

(1) Cette expression désigne les anmaux porteurs de la combinaison génétique susceptible de donner l'anomalie, mais ne l'exprimant pas. 


\author{
SUMMARY \\ HEREIJTARY PALATINE FISSURE ASSOCIATED \\ WITII LIMB ANCHYLOSIS IN THE " CHAROLAISE " BREED OF CATTLE
}

The palatine fissure associated with limb anchylosis sometimes noticed in the Charolaise breed of Cattle was studied on an anomalous calf and data were gathered from breeding and insemination centres.

The palate appeared fissured throughout and the anchylosis bore similary upon the knees, the hocks and fetlock joints. A thorough anatomical examination showed a shortening of hamstrings and the shifting and binding of some carpal and tarsal bones.

The scattered information from breeding stations and insemination centres entitle to stress the presence of unvarying phenomena. However, expressivity is not unalterable. Both sexes seem tobe equally touched. Calves were generally born alive but, since they were unable to stand up and feed properly because of the fissure, the breeders often slaughtered them as soon as born. know.

such associated congenital anomalies are not to be found in any other cattle breed as far as we

A certain number of data according to which, among the maternal grandsires of the anomalous calves were bulls whose offspring was palate-fissured and limb-stiffened could incline us to the view that the anomaly is governed by an autosomal recessive genre. However such an hypothesis does not account for the isolated cases recorded in the progeny of a number of bulls. Provided that these records are not due to errors in sires, which is rather frequent in the Charolaise breed, the hypothesis of an autosomal dominant gene with incomplete pentrance (20 per cent in the heterozygote) would better apply to all cases.

\title{
RÉFÉRENCES BIBLIOGRAPHIQUES
}

Kocil P., Fischer H., Scirumann H., I957. Libpalhologie der lanilwirlschaftlichen IIaustiere. Paul Parey, Berlin, $43^{6} \mathrm{pp}$.

MaLficot G., 1948. Les mathémaliques de lhérédité. Masson, Paris, $5^{8} \mathrm{pp}$.

Stang V., 1940. Gehäuftes Vorkommen von erblicher allyemeiner Gelenkverkrümmung in Verbindung mit Gaumenspalt beim Rind. Z. Tiersïchl. Zücht Biol., 46, 280-284. 